\title{
P38 Association of Daytime Augmentation Index with Brain-derived Neurotrophic Factor in Untreated Hypertensive Patients
}

\author{
János Nemcsik ${ }^{1, *}$, Dóra Batta ${ }^{1}$, Beáta Körösi ${ }^{1}, Z$ sófia Nemcsik-Bencze ${ }^{2}$, Andrea László ${ }^{3}$, Lilla Lénárt ${ }^{4}$, Andrea Fekete
}

${ }^{1}$ Semmelweis University, Department of Family Medicine, Budapest, Hungary

${ }^{2}$ Semmelweis University, Magnetic Resonance Research Center, Budapest, Hungary

${ }^{3}$ First German Hospital for Traditional Chinese Medicine, Bad Kötzting, Germany

${ }^{4}$ MTA-SE "Lendület" Diabetes Research Group Budapest, Budapest, Hungary

\begin{abstract}
Brain-derived neurotrophic factor (BDNF) is a neurotrophic molecule with impact on microvasculature and also with complex psychosomatic connections. The aim of our study was to evaluate its associations with 24 -hour brachial and central hemodynamic parameters and arterial stiffness in untreated patients who were studied because of elevated office blood pressure. Mobil-OGraph was used to measure the 24-hour brachial and central parameters. Serum BDNF was measured with ELISA, depression and anxiety were evaluated with questionnaires (Beck Depression Inventory and Hamilton Anxiety Scale, respectively). 73 patients were involved into the study. The average serum BDNF level was $28610 \pm 7965 \mathrm{pg} / \mathrm{ml}$. SeBDNF was correlated with daytime augmentation index (Aix, $r=0.234, p=0.046$ ). A borderline correlation was found with 24-hour total peripheral resistance $(r=0.227, p=0.053)$. In multiple linear regression model the association between Aix and BDNF remained significant after the adjustment for age, depression and anxiety $(B=0.243, p=0.034)$, but was attenuated after further adjustment for total vascular resistance $(B=0.186, p=0.106)$. In conclusions, the impact of BDNF on microvasculature can have physiological importance as through the mediation of total vascular resistance it can influence pulse wave reflection.
\end{abstract}

(c) 2019 Association for Research into Arterial Structure and Physiology. Publishing services by Atlantis Press International B.V. This is an open access article distributed under the CC BY-NC 4.0 license (http://creativecommons.org/licenses/by-nc/4.0/) 